\title{
Remote dynamic proxies for wave-based peer-to-peer haptic interaction
}

\author{
Zhi Li* \\ Department of Mechanical \\ Engineering \\ University of Victoria \\ Victoria, BC, Canada
}

\author{
Daniela Constantinescu ${ }^{\dagger}$ \\ Department of Mechanical \\ Engineering \\ University of Victoria \\ Victoria, BC, Canada
}

\begin{abstract}
This paper introduces a distributed haptic control architecture that can render direct interaction between users in addition to cooperative manipulation of virtual objects. The proposed architecture integrates remote dynamic proxies and peer-to-peer wave-based communications. Remote dynamic proxies are avatars of users at peer sites with motion governed by second order dynamics laws. They render physically-based motion of the distant users in the presence of update discontinuities caused by packet transmission limitations. They also enable users to touch their far away peers directly. The remote dynamic proxies are integrated with wave-based communications via a simplified symmetric algorithm for computing the transformation between wave variables and standard power variables. This algorithm implements peer-to-peer communications through input and output waves rather than master-slave communications through forward moving and returning waves. Wave variable controllers using this algorithm connect the distributed copies of the shared virtual object, and connect users to their remote dynamic proxies. The proposed control architecture is compared via experiments to peer-to-peer haptic cooperation with wave variable time delay compensation. The results illustrate that remote dynamic proxies with wave-based communications: (1) improve position coherency between the distributed copies of the shared virtual object; (2) render the mass of this object more faithfully in the presence of network delay; and (3) permit direct user-to-user interaction in addition to cooperative manipulation.
\end{abstract}

Index Terms: H.5.2 [Information Interfaces and Presentation]: User Interfaces-Haptic I/O; B.4.2 [Input/Output and Data Communications]: Input/Output Devices-Channels and controllers C.2.1 [Computer-Communication Networks]: Network Architecture and Design-Network communications

\section{INTRODUCTION}

Realistic force interaction between remote users connected via network is beneficial in a variety of areas, including surgical training [10], telerehabilitation [18], and computer games. Depending on the application, users involved in networked haptic interaction may need: (1) to manipulate virtual objects together; and (2) to touch and feel each other directly. For example, during surgical teletraining with force feedback, the expert surgeon and the remote resident may need to perceive each other's interaction with the virtual organ on which they operate. During haptics-based telerehabilitation, the therapist may need to guide and feel the hand of the remote patient directly in order to gauge their physical abilities.

Prior research addressing networked haptic interaction has focused primarily on cooperative manipulation of shared virtual objects. Both centralized (client-server) $[6,16]$ and distributed (peerto-peer) $[2,4,8,7,16,17]$ control architectures have been inves-

*e-mail: zhil@me.uvic.ca

†e-mail:danielac@me.uvic.ca tigated. Available studies have shown that peer-to-peer architectures can display larger contact stiffness [6] and can maintain higher position coherency between the copies of the shared virtual objects [16] than client-server architectures. In existing distributed approaches $[6,17]$, the remote users interact via their local copies of the shared virtual object. In turn, those local copies are connected via virtual coupling [5], time domain passivity [15] and wave variables [12] control. The investigation in [17] has shown that: virtual coupling control is sensitive to network delay; time domain passivity control may not be able to prevent distracting oscillations; wave variables control may enforce poor coherency between the distributed copies of the shared virtual object. In addition to connecting the local copies of the shared virtual object through virtual coupling, the architecture in [6] has provided the position and velocity (i.e., a kinematic proxy) of the remote user at the peer site. Although not developed for direct peer-to-peer interaction specifically, the architecture in [6] can render it.

Initial research into rendering direct user-to-user interaction has offered massless proxies with first order dynamics [10]. Compared to the conventional, purely kinematic proxies $[19,14]$, the motion of the dynamic proxies can be better controlled during collisions with fixed virtual objects or with other proxies. The performance of proxies with first order dynamics in networked haptic cooperation has not been investigated.

Recently, remote dynamic proxies with virtual coupling coordination [9] have been introduced for increasing the realism of networked haptic cooperation and for enabling direct force interaction between far away users. The remote dynamic proxies are avatars of users in the peer's virtual environment. They are governed by second order dynamics laws, and have been connected to their user via virtual couplers. The remote dynamic proxies have been shown to render smooth motion of the distant user regardless of update discontinuities due to low network transmission rates.

This paper proposes a distributed haptics control architecture that integrates remote dynamic proxies and peer-to-peer wavebased communications. It develops a simplified algorithm for computing the symmetric transformation between the wave and power domains [11, 17]. The algorithm distinguishes input and output waves rather than outgoing and returning waves. This classification allows the same transformation between the power and the wave variables to be used at all remote sites. Thus, it leads to the implementation of peer-to-peer communications rather than master-slave communications. Wave variable controllers based on this simplified algorithm connect the distributed copies of the shared virtual object, and connect users to their remote dynamic proxies. The proposed control architecture is compared via experiments to a recent peer-to-peer wave-based approach [17]. The results illustrate that remote dynamic proxies with wave-based communications: (1) improve coherency between the distributed copies of the shared virtual object; (2) render the mass of this object more faithfully in the presence of network delay; and (3) permit direct user-to-user interaction in addition to cooperative manipulation.

In the remainder of the paper, Section 2 introduces the remote dynamic proxy concept. Section 3 presents the simplified algorithm encoding the symmetric wave transformation. Section 4 overviews 
the peer-to-peer haptic architecture with remote dynamic proxies and wave-based communications. Section 5 compares the performance of the proposed architecture to the peer-to-peer scheme with wave-based delay compensation in [17]. Section 6 discusses conclusions and future work.

\section{THE REMOTE DYNAMIC PROXY}

This section introduces remote dynamic proxies for networked haptic interaction between two users. The extension to networked force cooperation among multiple users will be pursued in upcoming work. As in [9], remote dynamic proxies are proposed in order to enable far away users to touch and feel each other directly. Direct force interaction between distant users is expected to benefit physical therapists assisting remote clients.

A remote dynamic proxy is the avatar of a user in the virtual environment of their peer (see Figure 1, where RDP 12 denotes the remote dynamic proxy of Peer 1 in the virtual environment of Peer 2). The remote dynamic proxy inherits the inertial and damping properties from the haptic device of the user whom it represents. Its position and velocity are computed using physics-based simulation rather than being updated from network packets. The remote dynamic proxy is compliantly connected to the position and velocity transmitted by its user through wave-based communications. In Figure 1, the compliant connection between the user and their remote dynamic proxy is schematically represented via the spring $K_{\mathrm{T}}$ and damper $B_{\mathrm{T}}$ in the peer's virtual environment. This compliant connection allows the networked users to perceive the motion of distant peers smoothly regardless of update discontinuities due to network characteristics (e.g., limited bandwidth, delay, jitter, packet loss, etc.).

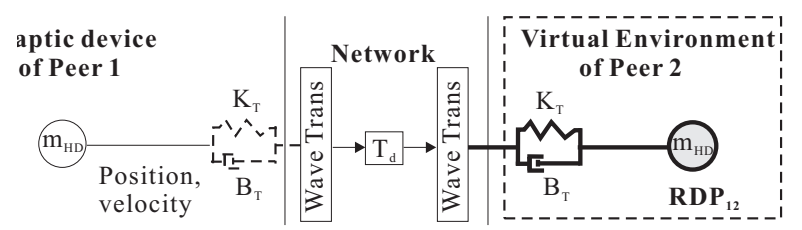

Figure 1: Remote dynamic proxy with wave-based communications.

The remote dynamic proxy is integrated into a peer-to-peer haptic control architecture with wave-based communications in Section 4. The integration employs the algorithm for encoding/decoding standard power variables into/from wave variables presented in the following section.

\section{Peer-to-Peer wave-based communications}

\subsection{Traditional symmetric configuration of wave vari- able control}

Wave (or scattering)-based communications have been introduced in teleoperation $[1,12]$ to render the communication line passive in the presence of communication delay. In teleoperation, the master and the slave robots play different roles. Their dissimilar functions have been embedded in control through distinguishing forward moving (from master to slave) and returning (from slave to master) waves. The two sides maintain distinct roles even when they communicate using the symmetric configuration [11] ${ }^{1}$.

Peer-to-peer haptic cooperation between two users via the symmetric configuration and the traditional wave taxonomy has been implemented in [17], as shown Figure 2. The figure details only the power-to-wave transformations and the virtual couplers coordinating the two peer copies of the shared virtual object. The virtual

\footnotetext{
${ }^{1}$ In the symmetric configuration, forces are encoded into, and velocities (and positions) are decoded from, wave signals at both sides.
}

coupling forces due to the interaction of each user with their local copy of this object provide the inputs in Figure 2. In this figure, notation is used as follows: indices 1 and 2 identify the two peers; $T_{\mathrm{d}}$ is the communication delay; $b$ is the wave impedance; $u$ and $v$ are the forward and returning waves, respectively; $K_{\mathrm{T}}$ and $B_{\mathrm{T}}$ are the gains of the coordinating controllers at the two remote sites; $F_{i}$ are the control forces on the copy of the shared object of Peer $i ; m_{\mathrm{O} i}$ and $b_{\mathrm{O} i}$ are the inertia and the damping of those copies; $x_{i}$ and $\dot{x}_{i}$ are their simulated position and velocity; and $\dot{x}_{i d}$ is their commanded velocity.

In the symmetric wave transformation shown in Figure 2, the control force on the copy of the shared virtual object of Peer 1 is:

$$
F_{1}=-B_{\mathrm{T}}\left(\dot{x}_{1 d}-\dot{x}_{1}\right)-K_{\mathrm{T}}\left(x_{1 d}-x_{1}\right),
$$

while the forward moving wave and the desired velocity are computed via:

$$
u_{1}=\frac{b \dot{x}_{1 d}+F_{1}}{\sqrt{2 b}}=v_{1}+F_{1} \sqrt{\frac{2}{b}} .
$$

and:

$$
\dot{x}_{1 d}=\frac{v_{1} \sqrt{2 b}+F_{1}}{b},
$$

After unwrapping the algebraic loop created by the combination of PD controller and the wave transformation, the desired velocity no longer depends on the control force (i.e., $F_{1}$ ):

$$
\dot{x}_{1 d}=\frac{v_{1} \sqrt{2 b}+B_{\mathrm{T}} \dot{x}_{1}+K_{\mathrm{T}}\left(x_{1}-x_{1 d}\right)}{b+B_{\mathrm{T}}}
$$

At the Peer 2 side, the control force, the returning wave and the desired velocity (after unwrapping the algebraic loop) are calculated using:

$$
\begin{gathered}
F_{2}=-B_{\mathrm{T}}\left(\dot{x}_{2}-\dot{x}_{2 d}\right)-K_{\mathrm{T}}\left(x_{2}-x_{2 d}\right), \\
v_{2}=\frac{b \dot{x}_{2 d}-F_{2}}{\sqrt{2 b}}=u_{2}-F_{2} \sqrt{\frac{2}{b}},
\end{gathered}
$$

$$
\dot{x}_{2 d}=\frac{u_{2} \sqrt{2 b}+B_{\mathrm{T}} \dot{x}_{2}+K_{\mathrm{T}}\left(x_{2}-x_{2 d}\right)}{b+B_{\mathrm{T}}} .
$$

Note that the asymmetric roles of the two teleoperation sides are reflected in the different definitions of the control forces $F_{1}$ and $F_{2}$. Furthermore, it is unclear how the traditional wave taxonomy can be employed to distinguish wave signals in distributed architectures that support interaction among multiple users. Therefore, the following section introduces a peer-based classification of wave signals.

\subsection{Peer-to-peer symmetric configuration of wave vari- able control}

The peer-based view of the haptic cooperation adopted in this work:

1. distinguishes outgoing (i.e., leaving a local site) and incoming (i.e., arriving at a local site) waves. Accordingly, $u_{\text {out } i}$ and $u_{\text {ini }}$ will hereafter identify the outgoing and incoming wave signals at Peer $i$, respectively (see Figure 3 ).

2. regards the haptic interfaces of all interacting users as similar to the master robot in teleoperation. Control forces at all peer sites will provide feedback to users in interaction with their local copies of the shared virtual object. Therefore, they are defined via:

$$
F_{i}=-B_{\mathrm{T}}\left(\dot{x}_{i d}-\dot{x}_{i}\right)-K_{\mathrm{T}}\left(x_{i d}-x_{i}\right) .
$$




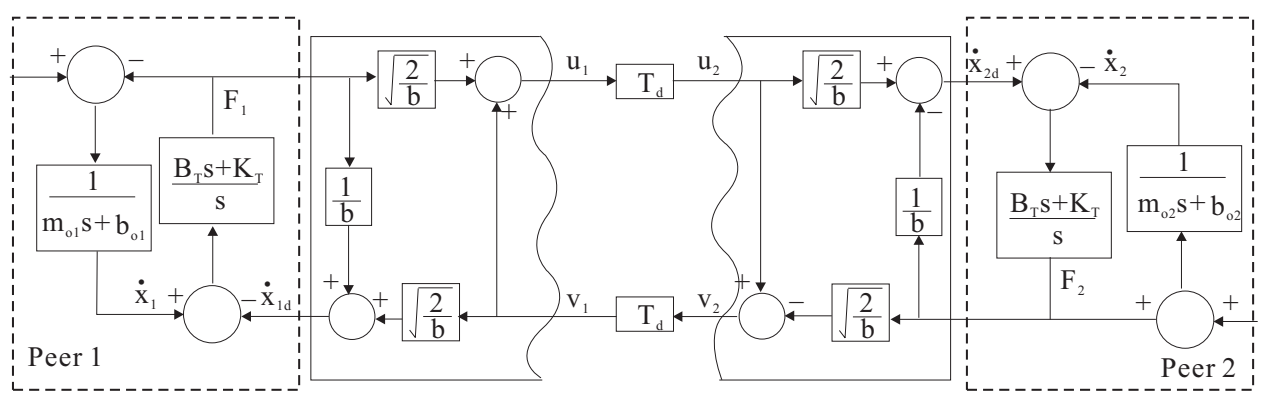

Figure 2: Traditional symmetric configuration of wave variable control of the shared virtual object [17].

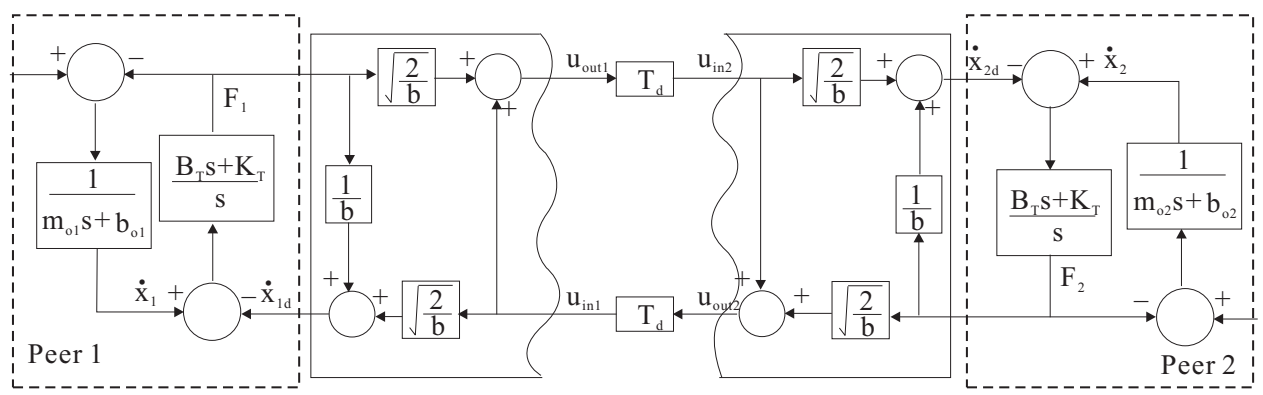

Figure 3: Peer-to-peer symmetric configuration of wave variable control of the shared virtual object.

Figure 3 illustrates the peer-to-peer symmetric configuration of wave variable control of the shared virtual object for haptic collaboration between two users.

Given the definition (8), the outgoing wave at Peer $i$ becomes:

$$
u_{\mathrm{out} i}=u_{\mathrm{in} i}+F_{i} \sqrt{\frac{2}{b}},
$$

while the desired velocity of the local copy of the shared object at Peer $i$ is computed (as in the traditional symmetric wave configuration) via:

$$
\dot{x}_{i d}=\frac{u_{\mathrm{in} i} \sqrt{2 b}+B_{\mathrm{T}} \dot{x}_{i}+K_{\mathrm{T}}\left(x_{i}-x_{i d}\right)}{b+B_{\mathrm{T}}} .
$$

To avoid drift of $x_{i d}$ in (10) (due to sampling, numerical integration, data loss, etc.), the communications are augmented to include wave integrals [13]. Then, the outgoing wave integral is computed via:

$$
U_{\text {out } i}=U_{\text {ini }}+p_{i} \sqrt{\frac{2}{b}},
$$

and the desired position is decoded using:

$$
x_{i d}=U_{\text {ini }} \sqrt{\frac{2}{b}}+\frac{1}{b} p_{i} .
$$

Notation in (11) and (12) follows [13], i.e., $U$ denotes wave integrals and $p$ is momentum (i.e., the integral of the control force):

$$
p_{i}=\int_{0}^{t} F_{i} d t .
$$

\section{DISTRIBUTED HAPTIC CONTROL WITH REMOTE DYNAMIC PROXIES AND WAVE-BASED COMMUNICATIONS}

The proposed distributed control architecture is shown in Figure 4 for haptic cooperation between two users. For simplicity, the two haptic devices are assumed similar. In Figure 4, notation is used as follows: $m_{\mathrm{HD}}$ and $b_{\mathrm{HD}}$ are the mass and the damping of the haptic interfaces; $m_{\mathrm{O} i}$ and $b_{\mathrm{O} i}$ are the mass and the damping of Peer $i$ 's copy of the shared virtual object; $K_{\mathrm{VC} i}$ and $B_{\mathrm{VC} i}$ are the stiffness and the damping of the contact between Peer $i$ and their copy of the virtual object; $K_{\mathrm{VC} i j}$ and $B_{\mathrm{VC} i j}$ are the stiffness and the damping of the contact between Peer $i$ 's remote dynamic proxy in Peer $j$ 's virtual environment and Peer $j$ 's copy of the virtual object; $x_{i}$ and $\dot{x}_{i}$ are the position and the velocity of the $i$-th haptic device; $x_{\mathrm{O} i}$ and $\dot{x}_{\mathrm{O} i}$ are the position and the velocity of Peer $i$ 's copy of the virtual object; $x_{i j}$ and $\dot{x}_{i j}$ are the position and the velocity of the remote proxy of Peer $i$ in the virtual environment of Peer $j ; x_{i d}$ and $\dot{x}_{i d}$ are the position and the velocity commands sent by the $i$-th haptic device to their peers via wave signals; $x_{\mathrm{Oid}}$ and $\dot{x}_{\mathrm{Oid}}$ are the position and velocity commands sent by Peer $i$ 's copy of the virtual object to the peer users; lastly, $F_{\mathrm{h} i}$ is the force applied by the $i$-th user to their device.

Note that, in the proposed architecture, the virtual environment of Peer $i$ comprises:

1. a copy of the virtual object jointly manipulated by the users.

2. the remote dynamic proxy $\mathrm{RDP}_{j i}$ of Peer $j$.

As in [17], the mass $m_{\mathrm{O}}$ of the shared virtual object is equally distributed between the local copies of this object, $m_{\mathrm{O} i}=\frac{m_{\mathrm{O}}}{2}$. Its damping $b_{\mathrm{O}}$ is assigned to each copy, $b_{\mathrm{O} i}=b_{\mathrm{O}}$.

The dynamics of the networked haptic cooperation rendered via the distributed architecture with remote dynamic proxies depicted in Figure 4 are:

- for the peer haptic devices:

$$
\begin{aligned}
& F_{\mathrm{h} 1}-F_{\mathrm{VC} 1}=m_{\mathrm{HD}} \ddot{x}_{1}+b_{\mathrm{HD}} \dot{x}_{1} \\
& F_{\mathrm{h} 2}-F_{\mathrm{VC} 2}=m_{\mathrm{HD}} \ddot{x}_{2}+b_{\mathrm{HD}} \dot{x}_{2}
\end{aligned}
$$

- for the remote dynamic proxies:

$$
\begin{aligned}
& F_{\mathrm{T} 12}-F_{\mathrm{VC} 12}=m_{\mathrm{HD}} \ddot{x}_{12}+b_{\mathrm{HD}} \dot{x}_{12} \\
& F_{\mathrm{T} 21}-F_{\mathrm{VC} 21}=m_{\mathrm{HD}} \ddot{x}_{21}+b_{\mathrm{HD}} \dot{x}_{21}
\end{aligned}
$$




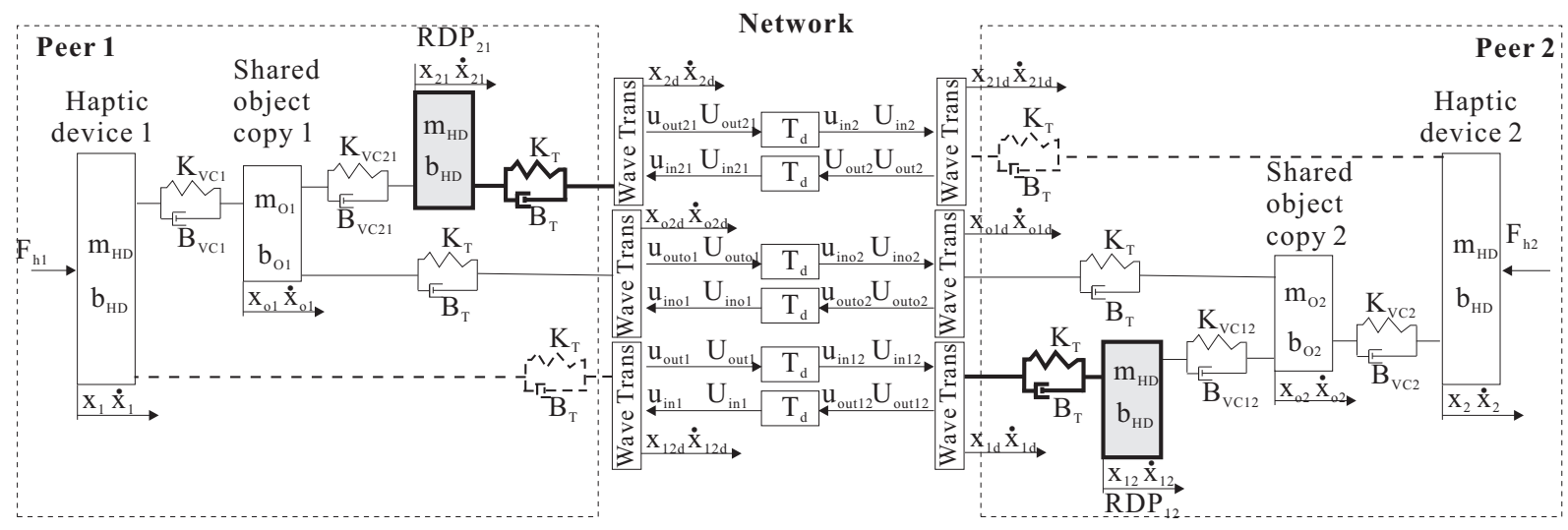

Figure 4: Peer-to-peer haptic cooperation with remote dynamic proxies and wave variable communications. The remote dynamic proxies are shaded, and their connection to the corresponding haptic device is bolded.

- for the local copies of the virtual object:

$$
\begin{aligned}
& F_{\mathrm{VC} 1}-F_{\mathrm{T} 1}+F_{\mathrm{VC} 21}=m_{\mathrm{O} 1} \ddot{x}_{\mathrm{O} 1}+b_{\mathrm{O} 1} \dot{x}_{\mathrm{O} 1} \\
& F_{\mathrm{VC} 2}-F_{\mathrm{T} 2}+F_{\mathrm{VC} 12}=m_{\mathrm{O} 2} \ddot{x}_{\mathrm{O} 2}+b_{\mathrm{O} 2} \dot{x}_{\mathrm{O} 2}
\end{aligned}
$$

where:

$$
\begin{gathered}
F_{\mathrm{VC} 1}=K_{\mathrm{VC} 1}\left(x_{1}-x_{\mathrm{O} 1}\right)+B_{\mathrm{VC} 1}\left(\dot{x}_{1}-\dot{x}_{\mathrm{O} 1}\right) \\
F_{\mathrm{VC} 2}=K_{\mathrm{VC} 1}\left(x_{2}-x_{\mathrm{O} 2}\right)+B_{\mathrm{VC} 1}\left(\dot{x}_{2}-\dot{x}_{\mathrm{O} 2}\right) \\
F_{\mathrm{T} 1}=K_{\mathrm{T}}\left(x_{\mathrm{O} 1}-x_{\mathrm{O} 2 d}\right)+B_{\mathrm{T}}\left(\dot{x}_{\mathrm{O} 1}-\dot{x}_{\mathrm{O} 2 d}\right) \\
F_{\mathrm{T} 2}=K_{\mathrm{T}}\left(x_{\mathrm{O} 2}-x_{\mathrm{O} 1 d}\right)+B_{\mathrm{T}}\left(\dot{x}_{\mathrm{O} 2}-\dot{x}_{\mathrm{O} 1 d}\right) \\
F_{\mathrm{VC} 12}=K_{\mathrm{VC} 12}\left(x_{12}-x_{\mathrm{O} 2}\right)+B_{\mathrm{VC} 12}\left(\dot{x}_{12}-\dot{x}_{\mathrm{O} 2}\right) \\
F_{\mathrm{VC} 21}=K_{\mathrm{VC} 21}\left(x_{21}-x_{\mathrm{O} 1}\right)+B_{\mathrm{VC} 21}\left(\dot{x}_{21}-\dot{x}_{\mathrm{O} 1}\right) \\
F_{\mathrm{T} 21}=K_{\mathrm{T}}\left(x_{2 d}-x_{21}\right)+B_{\mathrm{T}}\left(\dot{x}_{2 d}-\dot{x}_{21}\right) \\
F_{\mathrm{T} 12}=K_{\mathrm{T}}\left(x_{1 d}-x_{12}\right)+B_{\mathrm{T}}\left(\dot{x}_{1 d}-\dot{x}_{12}\right)
\end{gathered}
$$

The following section compares the Proposed Scheme (the peerto-peer control architecture with remote dynamic proxies and wavebased communications) to the Reference Scheme, i.e., the peerto-peer architecture with wave variable time delay compensation in [17]. The two schemes are contrasted via experimental networked haptic cooperation between two peers.

\section{EXPERIMENTS}

To ensure that successive networked cooperative interactions are comparable, controlled experiments are used to contrast the performance of the Proposed Scheme to the performance of the Reference Scheme. The human-applied force are replaced by preprogrammed forces applied to the FALCON NOVINT devices through commands sent to motors via software. Effectively, the inherent damping of the users' hands is eliminated from the interaction. However, the two haptic interfaces are impedance devices and thus, the controlled forces have no stabilizing effect compared to user-applied forces.

In all experiments, the shared virtual environment comprises a rigid cube in a rigid enclosure (see Figure 6). The enclosure is designed to ensure the same initial conditions among consecutive experiments and to restrict the manipulation to the $\mathrm{x}$-axis. The cube is transparent to permit visual evaluation of position coherency.

The experiments are conducted using the following contact and controller paramemters: $K_{\mathrm{VC} 1}=K_{\mathrm{VC} 2}=K_{\mathrm{VC} 12}=K_{\mathrm{VC} 21}=$ $4000 \mathrm{~N} / \mathrm{m} ; \quad B_{\mathrm{VC} 1}=B_{\mathrm{VC} 2}=B_{\mathrm{VC} 12}=B_{\mathrm{VC} 21}=3 \mathrm{Ns} / \mathrm{m} ; \quad K_{\mathrm{T}}=$
$2000 \mathrm{~N} / \mathrm{m} ; B_{\mathrm{T}}=200 \mathrm{Ns} / \mathrm{m}$. The mass of the virtual cube is $m_{\mathrm{O} 1}=$ $m_{\mathrm{O} 2}=0.5 m_{\mathrm{O}}=0.125 \mathrm{~kg}$, and the mass of the remote dynamic proxies is $m_{\mathrm{HD}}=0.1 \mathrm{~kg}$. Damping is incorporated neither in the remote dynamic proxies nor in the virtual cube, i.e., $b_{\mathrm{HD}=} b_{\mathrm{O} 1}=b_{\mathrm{O} 2}=$ $0 \mathrm{Ns} / \mathrm{m}$. The wave impedance is chosen to be $b=50 \mathrm{Ns} / \mathrm{m}$.

\subsection{Experimental setup}

Figure 5 illustrates the experimental networked haptic cooperation system. The system comprises two FALCON NOVINT haptic devices connected to two personal computers. One computer runs Window XP on an Intel Core 2 Duo CPU at $2.67 \mathrm{GHz}$ with $2 \mathrm{~GB}$ RAM. The other computer runs Window Vista on an Intel Core 2 Duo CPU at $1.67 \mathrm{GHz}$ with $3 \mathrm{~GB}$ RAM. The haptic devices provide 3 degrees of freedom (DOF) displacement sensing and force rendering and, thus, enable point interaction in 3DOF virtual environments. The computers are located in the same laboratory, and can be screened from each other to prevent users from seeing each other's display. Copies of a shared virtual environment comprising a rigid cube in a rigid enclosure are generated on each computer as $\mathrm{C}++$ console applications. The computers communicate over the network via the UDP protocol. A Wide Area Network Emulator (WANem) [3] simulates constant network delays, ranging from 0ms to $500 \mathrm{~ms}$. The WANem runs on a separate personal computer. The position sensing and force rendering rate of the FALCON NOVINT haptic devices is $1 \mathrm{KHz}$. The data transmission rate is $128 \mathrm{~Hz}$.

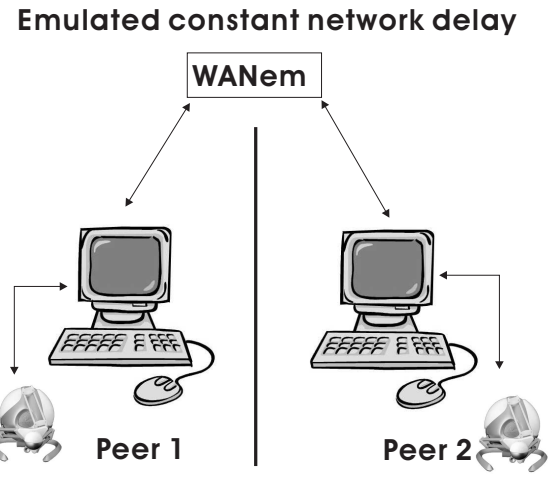

Figure 5: The experimental networked haptic cooperation system. 


\subsection{Experiment I - cooperative manipulation}

The first experiment investigates the networked cooperative manipulation of the virtual cube via the Proposed Scheme and via the Reference Scheme. Two measures are used to compare performance:

1. the position coherency between the two cube copies.

2. the error in rendering the mass of the cube.

Figure 6 is the snapshot of the screen of Peer 1 at the beginning of this experiment. Both users are initially at rest and in contact with the virtual cube. During the experiment, Peer 1 pushes both the virtual cube and Peer 2 with a constant force $F_{\mathrm{h} 1}=3 \mathrm{~N}$ while Peer 2 applies no force.

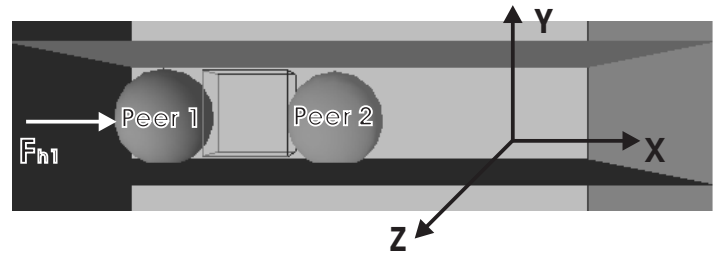

Figure 6: Snapshot of the beginning of Experiment I (cooperative manipulation) at Peer 1 .

Figure 7 plots the position coherency during networked cooperation both via the Proposed Scheme and via the Reference Scheme, for network delays of $50 \mathrm{~ms}$ and $200 \mathrm{~ms}$, respectively. The data in this figure demonstrate that the Proposed Scheme (peer-to-peer architecture with remote dynamic proxies and wave-based communications) coordinates the two copies of the virtual cube better than the Reference Scheme (peer-to-peer architecture with wave variable delay compensation).

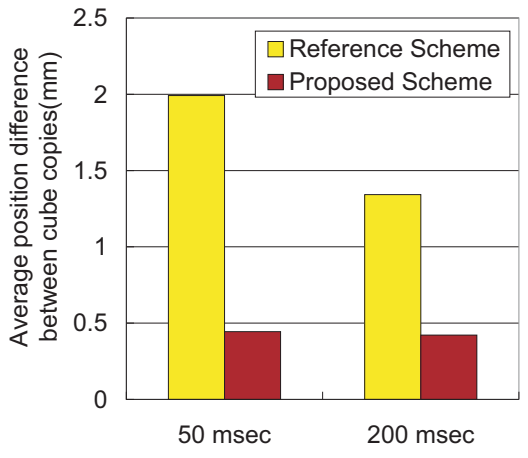

Figure 7: Position coherency for haptic cooperation via the Reference and via the Proposed schemes.

Figure 8 shows the positions of the virtual cube at each peer, both for the Proposed Scheme and for the Reference Scheme, for network delays of $50 \mathrm{~ms}$ and $200 \mathrm{~ms}$, respectively. These parabolic experimental trajectories demonstrate that both schemes render the virtual cube to Peer 1 as a pure mass in free space (no damping is assigned to the cube or the remote dynamic proxies in this experiment). Hence, Peer 1 perceives physically-based dynamics of the virtual cube when wave variable controllers are used to connect the peer sites. However, Figure 8 also illustrates that the mass perceived by Peer 1 depends on the control architecture and on the network delay. Figure 9 plots the cube mass rendered by the Proposed
Scheme and by the Reference Scheme for four network delays. This figure demonstrates that the Proposed Scheme reders the cube mass more faithfully than the Reference Scheme for all network delays.

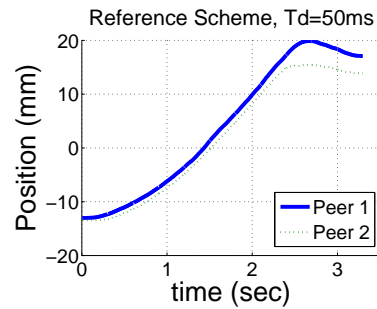

(a)

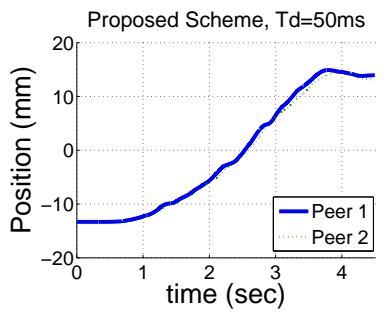

(c)

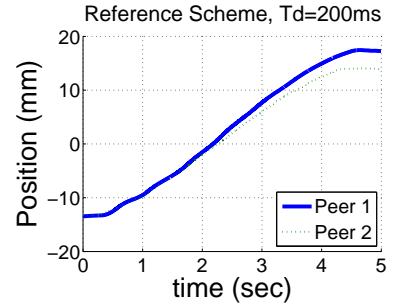

(b)

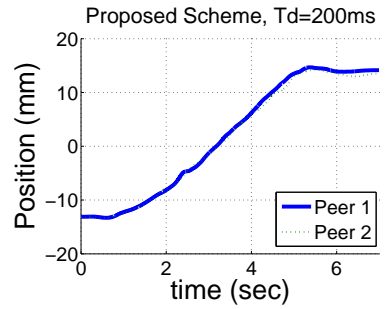

(d)
Figure 8: Experimental positions of the virtual cube at each peer.

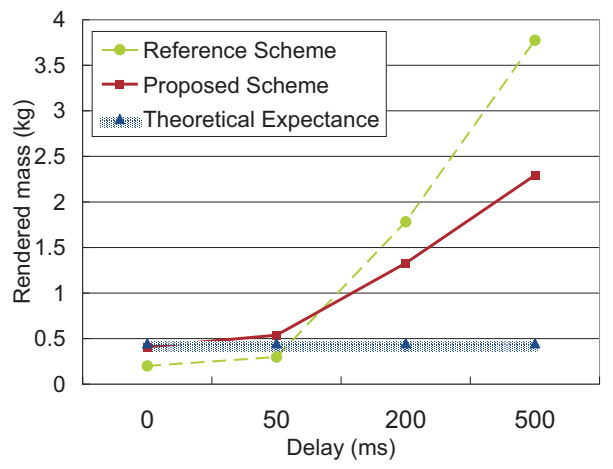

Figure 9: Mass of the virtual cube: rendered to Peer 1 via the Reference and via the Proposed schemes; simulated (Theoretical).

\subsection{Experiment II - user-to-user contact}

The second experiment shows that remote users can touch each other when they are connected via the Proposed Scheme. Direct user-to-user contact cannot be rendered via the Reference Scheme.

Figure 10 is the snapshot of the screen of Peer 1 at the beginning of this second experiment. The two users are initially at rest and in contact with each other. During the experiment, Peer 1 pushes Peer 2 with constant force $F_{\mathrm{h} 1}=3 \mathrm{~N}$. Peer 2 applies no force to Peer 1. A constant network delay $T_{\mathrm{d}} \mathrm{ms}$ is emulated via the WANem.

The positions of the two peers and the forces applied to them are depicted in Figure 11. Note that both users are in continuous and smooth contact with their peer throughout the experiment. The data in Figure 11 validate that the proposed peer-to-peer architecture with remote dynamic proxies and wave-based communications 


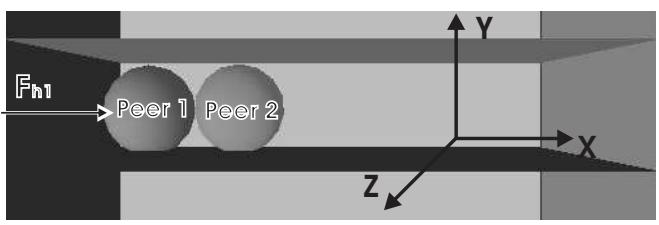

Figure 10: Snapshot of the beginning of Experiment II (user-to-user contact) at Peer 1.

is suitable for rendering user-to-user contact in addition to cooperative manipulation of shared virtual objects.

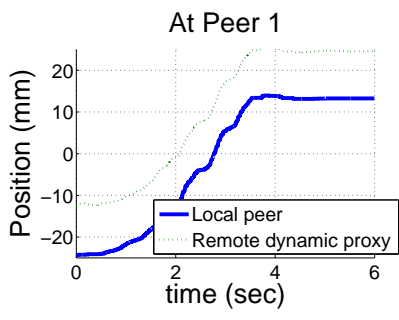

(a)

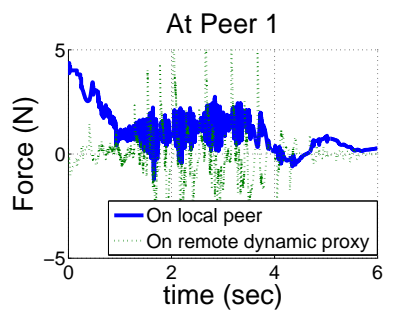

(c)

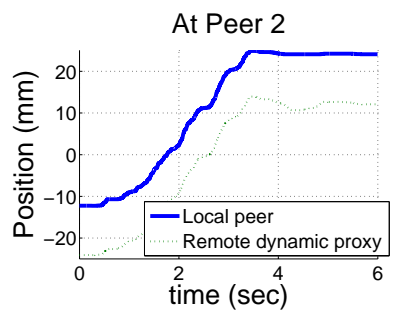

(b)

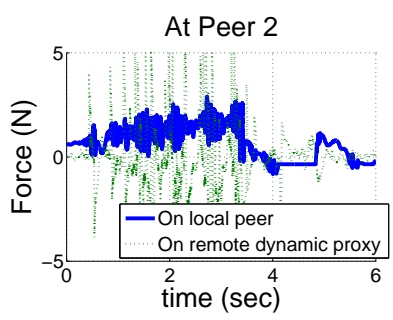

(d)
Figure 11: Experimental positions and forces during direct user-touser interaction.

\section{CONCLUSIONS AND FUTURE WORK}

This paper has introduced a distributed control architecture for networked haptic cooperation. The proposed architecture integrates remote dynamic proxies and peer-to-peer wave-based communications. The remote dynamic proxies are avatars of users at peer sites with motion governed by second order dynamics laws. They render physically-based motion of the distant users in the presence of update discontinuities caused by packet transmission limitations. Wave variable controllers connect the distributed copies of the shared virtual object, and connect users to their remote dynamic proxies. These controllers implement a peer-to-peer algorithm for encoding/decoding standard power variables into/from wave variables. The proposed distributed architecture has been compared via experiments to peer-to-peer haptic collaboration with wave variable time delay compensation. The experimental results have illustrated that remote dynamic proxies with wave-based communications: (1) improve position coherency between the distributed copies of the shared virtual object; (2) render the mass of this object more faithfully in the presence of network delay; and (3) permit direct userto-user interaction in addition to cooperative manipulation.

Upcoming work focuses on the stability analysis of the proposed networked haptic cooperation architecture, as well as on the comparative investigation of the performance of remote dynamic proxies integrated with various coordination controllers (eg., virtual couplers).

\section{ACKNOWLEDGEMENTS}

This work was supported through an NSERC Discovery Grant.

\section{REFERENCES}

[1] R. Anderson and M. Spong. Bilateral Control of Teleoperation with Time Delay. IEEE Trans. Autom. Control, 34(5):494-501, 1989.

[2] P. Buttolo, R. Oboe, and B. Hannaford. Architectures for Shared Haptic Virtual Environments. Computer \& Graphics, pages 1-10, 1997.

[3] P. E. R. Centre. WANem 1.1 Wide Area Network Emulator User Guide. 1:1 - 15, 2007.

[4] J. Cheong, S.-I. Niculescu, A. Annaswamy, and M. Srinivasan. Motion synchronization in virtual environments with shared haptics and large time delays. In World Haptics 2005, pages 277-282, 2005.

[5] J. Colgate and M. Stanley. Issues in the Haptic Display of Tool Use. In IEEE/RSJ Int Conf Intell Robot Syst, pages 140-145, Pittsburgh, PA, 1995.

[6] M. Fotoohi, S. Sirouspour, and D. Capson. Stability and Performance Analysis of Centralized and Distributed Multi-rate Control Architectures for Multi-user Haptic Interaction. Int J Robot Res, 26(9):977994, 2007.

[7] M. Glencross, C. Jay, J. Feasel, L. Kohli, M. Whitton, and R. Hubbold. Effective Cooperative Haptic Interaction over the Internet. In Virt Real Conf, 2007. VR '07. IEEE, pages 115-122, Manchester Univ., 2007.

[8] J. Kim, H. Kim, B. Tay, M. Muniyandi, M. Srinivasan, J. Jordan, J. Mortensen, M. Oliveira, and M. Slater. Transatlantic Touch: A Study of Haptic Collaboration over Long Distance. Presence, 13(3):328-337, 2004

[9] Z. Li and D. Constantinescu. Networked Haptic Cooperation using Remote Dynamic Proxies. In submitted to the Int Conf Adv CompHuman Interf, 2009.

[10] P. Mitra and G. Niemeyer. Dynamic Proxy Objects in Haptic Simulations. In IEEE Conf Robot Autom Mechatronics, pages 1054-1059, 2004.

[11] G. Niemeyer. Using wave varialbes in time delayed force reflecting teleoperation. $\mathrm{PhD}$ thesis, Massachusetts institute of technology, 1997.

[12] G. Niemeyer and J. E. Slotine. Stable Adaptive Teleoperation. IEEE J. Oceanic Eng., 16(1):152-162, 1991.

[13] G. Niemeyer and J. E. Slotine. Telemanipulation with Time Delays. Int. J. Robot. Res., 23(9):873-890, 2004.

[14] D. Ruspini, K. Koralov, and O. Khatib. Haptic Interaction in Virtual Environments. In IEEE/RSJ Int Conf Intell Robot Syst, Genoble, France, 1997.

[15] J.-H. Ryu and C. Preusche. Stable Bilateral Control of Teleoperators Under Time-varying Communication Delay: Time Domain Passivity Approach. In IEEE Int Conf Robot Autom, pages 3508 - 3513, 2007.

[16] G. Sankaranarayanan and B. Hannaford. Experimental comparison of internet haptic collaboration with time-delay compensation techniques. In Symp Haptic Interf Virt Envir Teleop Syst, pages 259 - 266, 2008.

[17] G. Sankaranarayanan and B. Hannaford. Experimental comparison of internet haptic collaboration with time-delay compensation techniques. In IEEE Int Conf Robot Autom, pages 206-211, 2008.

[18] H. Sugarman, E. Dayan, A. Weisel-Eichler, and J. Tiran. The Jerusalem Telerehabilitation System, a New, Low-Cost, Haptic Rehabilitation Approach. CyberPsychology \& Behavior, 9(2):178-182, 2006.

[19] C. Zilles and J. Salisbury. A Constraint-based God Object Method for Haptic Display. In ASME Haptic Interf Virt Envir Teleop Syst, pages 146-150, Chicago, IL, 1994. 